\title{
CURRENT TRENDS IN THE LABORATORY DIAGNOSIS OF TUBERCULOSIS
}

\section{*E. Ogbaini-Emovon}

*Department of Medical Microbiology,University of Benin Teaching Hospital, Benin City, Nigeria.

\section{Correspondence:}

Dr E. Ogbaini-Emovon

Department of Medical Microbiology

University of Benin Teaching Hospital,

Benin City, Nigeria.

E-mail: ephogbaini@yahoo.com

\section{INTRODUCTION}

Mycobacterium tuberculosis (M. tuberculosis) causes tuberculosis and is a very important pathogen of humans. Tuberculosis kills more people than ever, with the increasing number of HIV-infected individuals ${ }^{1}$. The fact sheet of global tuberculosis is stunning with one third of the world's population currently infected and new infections occur every second, resulting in new tuberculosis infection in $1 \%$ of the world population annually $^{2}$. It is projected that newly acquired infections between 2002 and 2010 would be 1 billion persons, of these, 150 million will get sick and 36 million will die of tuberculosis ${ }^{2}$. In SubSahara African countries, the incidence of tuberculosis has doubled since the early $1980 \mathrm{~s}^{3}$.

Mycobacterium tuberculosis is related and associated with other species of mycobacteria, referred to as the Mycobacteria Tuberculosis Complex (MTB complex). MTB complex includes; $M$. bovis, (including the vaccination strain $B C G) \quad M$. tuberculosis, M. africanum, M. microti, and $M$. canettii ${ }^{4}$. These grouping is based on several parameters which include the analysis of antigenic extracts, target epitopes for monoclonal antibodies and antigenic and DNA relatedness ${ }^{5}$.
Historically, the diagnosis and treatment of $M$. tuberculosis has been difficult, because of its peculiarities; it has a high concentration of lipids in the cell wall, which makes it impermeable to stains and dyes, requiring special staining techniques for microscopic observation, resistant to many antibiotics, resistant to osmotic lysis via complement deposition, as well as resistant to lethal oxidation and survival inside macrophages, making it possible to undergo a long period of latency. Unlike many pathogenic bacteria, M. tuberculosis also grows slowly, making culture and susceptibility testing time consuming. This write- up examines the critical role of the clinical microbiology laboratory, through the various modalities of laboratory diagnosis, strain-typing and susceptibility testing, with emphasis on the advantages and drawback of these techniques.

\section{ROLE OF THE MEDICAL MICROBIOLOGY LABORATORY}

The laboratory physicians have been on the front line in the fight against tuberculosis (TB) ever since Dr Robert Koch announce his discovering of the tuberculus bacillus in 1882 . Driven by the worldwide increase in the incidence of tuberculosis, several changes in laboratory practice have 
evolved over the past several years. The challenge for the clinical laboratory is to provide the most rapid and reliable technologies for diagnosis and antimycobacterial drug susceptibility, as well as speciation and strain- typing, possibly within days or even hours rather than weeks.

\section{MODALITIES OF LABORATORY DIAGNOSIS}

1) Microscopy

2) Culture

3) Immunodiagnosis

4) Molecular Techniques

\section{CLINICAL SPECIMENS}

This depends on the clinical presentation and may include any body fluid or tissue ${ }^{6}$

\section{1) Pulmonary Tuberculosis}

This is the commonest form of tuberculosis and various respiratory specimens may be useful, including ; spontaneously expectorated sputum, normal - saline nebulize induced sputum transtracheal aspirate, bronchoalveolar lavage, bronchoalveolar brushing etc.

\section{2) Extra} Tuberculosis

pulmonary

This may affect various organs of the body and as such the following body fluids and tissues may be useful depending on clinical presentation.; pleural fluid ,pericardial fluid, peritoneal fluid, urine, joint aspirate, gastric aspirate, CSF, pus, blood, bone marrow biopsy or aspirate, lymph node, skin and bone.

\section{Collection, Transport and Storage of Specimen}

\section{Sputum:}

Three specimen of sputum are required in 2 visits for two days; Spot -
Morning -Spot. However, for patients on admission, the specimens can conveniently be collected for three days, with at least one as morning sample. A sterile universal container that is leak-proof is required. Patient is instructed to cough out deeply from the chest. Saliva is unacceptable. A good sputum is thick and purulent. Specimen is transported immediately to the laboratory in a sealed plastic bag. Transport within 2 hours or refrigerate if delay is expected .For gastric aspirate, a calculated amount of $\mathrm{Na}_{2} \mathrm{CO}_{3}$ is added if delay for more than 2 hours is anticipated. Blood and CSF are not to be refrigerated.

\section{Tissues and Body fluids:}

Generally tissue specimens are collected by biopsy and body fluid by invasive techniques. Tissue specimen and needle biopsy material should be placed in a small quantity of broth such as Middlebrook $7 \mathrm{H} 9$ or $7 \mathrm{H} 11$ as a holding medium and sent to the medical microbiology and or histopathology laboratory for analysis

\section{Stool:}

Stool specimen may be valuable in patients with AIDS, as the concentration of mycobacteria especially Mycobacteria avium intercellularis (MAI) may be sufficiently high in the lower intestinal tract to be recovered in culture ${ }^{7}$

\section{Urine:}

Urine specimen may be useful in the diagnosis of renal tuberculosis. At least three early morning urine specimens should be collected on successive 24-hour periods to maximize the chance of recovery of mycobacterium.

\section{SAFETY CONSIDERATIONS}

Generally, Mycobacterium tuberculosis is highly hazardous,stringent safety 
control standards conforming to the Biosafety Level 3 (BSL 3) with a Biosafety Cabinet 2(BSC 2) and possibly a dedicated laboratory is required for specimen processing ${ }^{5}$.

\section{1) Microscopy}

This is by far the most widely applied diagnostic technique for tuberculosis. It is a key test in both diagnostic and establishing infectiousness. In most resource poor setting, it is the only available option. Microscopy can be carried out on virtually all clinical specimens and requires a simple microscope or fluorescent type and few reagents ${ }^{8}$. Microscopy is usually the first line test performed on specimen. It can be simple microscopy using Ziehl- Neelson Staining technique or fluorescent microscopy using fluorochrome such as auramine phenol to enhance visualization. Turnaround time is less than 2 hours.

Advantages of Microscopy: It is cheap, rapid and specific.

Disadvantages of Microscopy: It is subjective, less sensitive-about 30$70 \%$ which requires at least 5000 10,000 AFB per $\mathrm{ml}$ of sputum for positive result ${ }^{8}$. Requires 3 sputa and is labour intensive. Fluorescent microscopy is more sensitive than simple microscopy ${ }^{9}$. The requirement for a fluorescent microscope makes it prohibitive. Concentration of specimen by centrifugation can increase sensitivity for non sputum specimen.

False Positive: The following if present in a specimen can result in a false positive result: Norcadia $s p p$, Gordonia spp., Tsukamurella spp. Legionella micdadei, bacteria endospores, Rhodococcus spp (sputum), M. Gastri (Gastric aspirate), M. Smegmatis (Urine),M. Gordonae (Water Contamination), artefactual scratch on the slide.

\section{Determination of infectiousness in a patient with pulmonary tuberculosis}

If sputum sample from a previously positive patient are negative on 3 consecutive occasions over 14 days or a duration proportionate to the effectiveness of the drug treatment Patient can be assumed to be non infectious by the respiratory route.

\section{3) Culture}

This is the gold standard for the laboratory diagnosis of tuberculosis. A definitive diagnosis of TB requires identification of $M$. tuberculosis from a specimen

Indications for culture: Confirmation of Microscopy when uncertain, treatment failure, relapse,monitoring of Multidrug Resistance (MDR), identification of mycobacterium variants e.g $M$. boris for epidemiological purposes and for antimicrobial sensitivity testing. It is more sensitive than microscopy - able to detect $10-100$ viable organism per $\mathrm{ml}$ of specimen ${ }^{8}$. Before culture, sputum require decontamination and digestion with 2-4\% NaOH- NALC solution (N-acetyl, L-cystein). Decontamination kills all the normal flora of the respiratory tract capable of overgrowing in the culture media. Digestion is to dissolve mucin and other organic component of sputum that may wall off the bacilli from accessing the nutrient of the media. Specimen from sterile sites does not require decontamination. Concentration by centrifugation is required for such specimen before culture.

Media- The common media for the culture of mycobacterium tuberculosis can be classified into three categories:

(a) Egg-based media such as Lowenstein - Jensen (LJ) medium. Duration of culture is 2-6 weeks. 
(b) Agar- based media such as Middlebrook 7H10 and 7H11.These requires about 3-4 week.

(c) Liquid Media such as Middlebrook 7H12 and 7H13. These can yield result within few days.

Advantages of culture: Very sensitive, can be used for drug sensitivity testing, and for characterization of species.

Limitations of culture: Requires characterization for species identification, very slow-takes some week

\section{BACTEK RAPID RADIOMETRIC CULTURE SYSTEM}

In 1980, Becton Dickson introduced the BACTEC 460 TB Radiometric System. This uses liquid media, Middle brook $7 \mathrm{H} 12$ or $7 \mathrm{H} 13$ which contain 14C - labelled palmitic acid. Growing mycobacteria utilizes the acid releasing radioactive $\mathrm{CO} 2$ which is measured by BACTEC instrument which indicate it as "growth index"

Advantages: BACTEC method can be read less than 7 days of incubation, averages 9-14 days. There is also an increase in the yield of positive cultures from clinical specimens compared with conventional solid media $^{10}$. It can be adapted for antimicrobial sensitivity testing ${ }^{11}$.

Limitations: It cannot be used for colonial morphology in organism identification, cost of instrumentation and the need for disposal of radioactive material

\section{BACTEK \\ MYCOBACTERIA GROWTH INDEX TUBE (MGIT)}

The MGIT is a nonradiometric, flourescence, manual or automated system for the rapid detection of mycobacterium species from various sites of the body. It consist of a glass tube with modified $7 \mathrm{H} 12$ broth base, enriched with basic nutrients for growth of mycobacteria and a mixture of antibiotics to inhibit the growth of contaminating bacteria. A flourescent compound is embedded at the bottom of the tube. The flourescent compound does not flourence in the presence of oxygen, but following depletion of oxygen as a result of mycobacteria growth there is flourescence which can be detected visually under ultraviolet light. In the automated version (MGIT 960 system), flourescence is detected by a sensor. The system holds 960 tubes which are continuously monitored.

\section{LYSIS-CENTRIFUGATION BLOOD CULTURE SYSTEM}

The recovery of mycobacterium from peripheral blood and bone marrow samples may be improved by releasing the intracellular mycobacterial cells into the blood culture broth, increasing the rate and reducing the time of recovery. In the lysis- centrifugation blood culture method, blood is put into a tube containing an anticoagulant and a lysing agent to effect rupture of both erythrocytes and neutrophils. Following centrifugation of the tube, the sediment is inoculated into the appropriate culture media. This method has increased both the yield and shortened the time of recovery of mycobacteria from blood cultures ${ }^{12,13}$.

\section{PHAGE TECHNIQUE (PAT)}

AMPLIFICATION

This is a bacteriophage - based test to detect $M$. tuberculosis in sputum. Patient sputum is mixed with a reagent which contains mycobacteriophage. The bacteriophage infect the mycobacterium tuberculosis in the sputum and multiply within it. A virucide is added which destroys any mycobacteriophage that remains outside the tubercle bacilli. The 
bacteriophages after multiplication lyse the mycobacterium cells and are released. Rapid (overnight) growing non-pathogenic mycobacteria (sensor cells) which can also be infected by the phage are added and the sample is incorporated in an agar mixture, plated and incubated overnight at $37^{\circ} \mathrm{C}$. The phage replicate, infect and lyse the sensor cells leaving zones of clearing (holes) in the agar. These areas of clearing indicates that the patient sputum contain viable $M$. tuberculosis.

Advantages of PAT: It is fast with a turnaround time of 2 days. Cheap and requires few equipments. Sensitivecan detect as low as 100 tubercles per $\mathrm{ml}$ of sputum ${ }^{5}$. It can be adapted for sensitivity testing.

Limitations of PAT: Applicable to sputum specimen only and technically demanding.

4) IMMUNOLOGICAL METHODS Immunological tests are of limited application due to cross reactivity and poor sensitivity Examples are the Myco Dot antibody test,tuberculin skin test and the interferon gamma assay. Myco Dot Antibody Test:

It uses purified lipoarabinomannam as antigen. Lipoarabinomannan is a glycolipid found in the cell wall of mycobacteria species. In this approach, the antibody produced in response to the mycobacterium infection is detected in the patient serum by immunoassay.

Advantages: Very quick, as fast as 20 minutes and easy to perform.

Limitations: Sensitivity is low, can only detect high levels of antibody as found in active diseases. It does not rule out TB in patients with poor antibody response as in HIV and malnutrition. Not specific due to cross reactivity with other species of mycobacteria in the environment such as the Non-Tuberculous
Mycobacterium (NTM) which the individual is exposed to.

\section{TUBERCULIN SKIN TEST (MANTOUX TEST)}

The tuberculin test such as mantoux test, is a diagnostic tool for tuberculosis. It is clinically used as a screening test. Nearly 100 years old and still the standard of practice in the United State in the diagnosis of latent TB infection. In this test, a standard dose of 5 tuberculin units $(0.1 \mathrm{ml}$ of a Purified Protein Derivative (PPD) is injected intradermally and read 48$72 \mathrm{hrs}$ later. A person who has been exposed to the bacteria is expected to mount an immune response in the skin containing the bacteria protein (PPD). The reaction is read by measuring the diameter of induration (palpable raised hardened area across the forearm) in millimeters. Erythema (redness) should not be measured. If a person has had a history of a positive tuberculin test, another test is not needed. But if negative, another test may be needed.

Interpretation: The result of this test must be interpreted carefully. The person's medical risk factors determine at which increment $(5 \mathrm{~mm}$, $10 \mathrm{~mm}$ or $15 \mathrm{~mm}$ ) of induration the result is considered positive. A positive result indicates TB exposure.

\section{CDC CLASSIFICATION OF} TUBERCULIN TEST

The Centre for Disease Control USA bases interpretations on individual medical risk factors as follows;

$5 \mathrm{~mm}$ or more :- Positive for the following categories of individual; HIV positive patient, recent contact of TB case, Patient with nodular or fibrotic changes on CXR - evidence of old healed TB, patient with organ transplant with Immuno-compromise.

$10 \mathrm{~mm}$ or more: positive for recent arrivals (< 5yrs) from high prevalent countries, injection drug users, 
residents and employee of high risk settings eg Mycobacteriology laboratory personnel, children < 4 years or adolescent exposed to adult in high risk category.

15mm or more: Positive for person with no known risk factor for TB

False Positive Results: This may be observed in the following circumstances; infection by nontuberculous mycobacteria (NTM).e.g mycobacterium avium intercellularis (MAI), from the enviroment, water and food, especially

immunocompromised patients.

Previous administration of BCG vaccine. This may result in false positive results for many years afterward $^{14}$.

False Negative Results: This may result from conditions such as anergy which is the lack of the body's defence mechanisms when it comes into contact with foreign substances. eg AIDS and also in overwhelming TB when the immune response has waned considerably.

\section{BCG AND TUBERCULIN TEST}

There is disagreement on the use of tuberculin test on people who have been immunized with BCG. The US recommendation is that previous $B C G$ should be ignored, while in the UK, the recommendation is that interferon tests should be used to help interpret positive tuberculin test. The US approach is likely to lead to more false positive and more unnecessary treatment with potentially toxic drugs.

\section{INTERFERON-GAMMA RELEASE ASSAYS (IGRA)}

Because of the difficulties with the tuberculin test interpretation, the interferon-gamma assay test was developed. This test is used in all circumstances in which tuberculin test is currently being used such as contact investigations, evaluation of recent immigrants who have had BCG vaccination, TB screening of health care workers etc. Two available formats of the interferon-gamma release assays are; the QuantiferonTB Gold and T Spot-TB test. The IGRA assay is based on the ability of the Mycobacterium tuberculosis antigens, which includes the Early Secretory Antigen Target 6 (ESAT-6) and Culture Filtrate Protein 10 (CFP10) to stimulate host production of interferon-gamma. Because these antigens are not present in NTM or in BCG vaccine, these tests can distinguish latent tuberculosis infection from BCG immunization. The Quantiferon- TB Gold was approved by the US Food and Drug Administration (FDA) in 2005. This is a whole blood test. Blood samples are mixed with synthetic peptides of ESAT- 6 and CFP-10 and incubated for 16-24 hrs, the amount of interferongamma released by the lymphocytes is detected by enzyme immunoassay. The $T$ Spot-TB test uses a different method of detecting interferon-gamma from peripheral mononuclear cells. In $T$ Spot-TB assay, T-cells releasing interferon-gamma to ESAT-6 and CFP-10 are detected and quantified as spot-forming cells.

Numerous studies that have been conducted throughout the world using these methods show higher specificity and better correlation with exposure to M. tuberculosis ${ }^{15-19}$. However, clinical evaluation and additional tests such as chest radiograph, sputum smear and culture are required to confirm the diagnosis of latent TB infection or TB disease.

Advantages: Requires a single visit to draw a blood sample and result available within $24 \mathrm{hrs}$.It does not boost immune response measured by subsequent tests which can happen with tuberculin skin test. Not subject to 
readers bias as in tuberculin skin test and not affected by prior BCG vaccination ${ }^{20}$.

Disadvantages: Blood must be processed within $12 \mathrm{hrs}$ while WBC are still viable. There are limited data on use in children younger 17 yrs of age and immunocompromised patients e.g HIVIAIDS, diabetics, treatment with immunosuppresive drugs In the diagnosis of active TB, the interferon gamma release assays have reduced sensitivity due to their ability to detect latent $\mathrm{TB}^{20}$.

Presently a version of Quantiferon - TB Gold test called Quantiferon-TB Gold In-Tube is now available in Europe, which can provide direct antigen stimulation in the blood-drawing tubes and eliminate the maximum 12-hours processing requirement ${ }^{21}$.

In general the adoption of these tests (IGRA), requires a dramatic shift, as the diagnosis of TB infection will be made in the laboratory instead of the clinic. This is a welcome shift in that laboratories are more likely to give reliable results because of routine quality assurance and proficiency testing, something that is not possible in all clinical settings in a given locale.

\section{4) MOLECULAR DIAGNOSTIC TECHNIQUES (DNA PROBE AND PCR)}

Molecular techniques are aimed at the nucleic acid of the mycobacterium as the analyte.They are the newest diagnostic techniques in the armamentarium of clinical microbiology laboratory and include techniques such as DNA Probe and the Polymerase Chain Reaction $(P C R)^{22}$. Ribosomal rRNA is useful genetic target for the identification of organisms, since it often contain signature sequences and is present in the cells and culture in high quantity secondary to the growth of the bacillus (biologic amplification).
There are various applications of these in the detection and identification of Mycobacterium tuberculosis ${ }^{23-26}$. These includes; culture confirmation of isolates recovered from clinical specimens using DNA probes, the identification of Mycobacteria through DNA sequencing, direct detection of M. tuberculosis in clinical specimen using nucleic acid amplification assays e.g Polymerase Chain Reaction (PCR) and DNA fingerprinting and straintyping of Mycobacterium species. The concern is that these state-of-the art technologies are complex and expensive and are not affordable in developing countries where $95 \%$ of the global burden of tuberculosis is located.

Advantages of Molecular Techniques: They are rapid (within few hours), highly sensitive and specific. Disadvantages of Molecular Techniques: Expensive, requires expertise and may not differentiate active infection as DNA from a dead organism during antibiotic treatment can be detected and amplified by PCR.

\section{SPECIATION AND STRAIN-TYPING}

This is important for purposes of epidemiologic studies and may help to establish new infections or mutant strains as well as determination of sources of infection e.g Mycobacterium bovis from infected milk source, patient with multidrug resistant TB (MDR-TB) etc. Straintyping can expand our understanding of transmission, including in settings outside the traditional concentric-circle approach, where TB transmission can occur. Once an isolate has been recovered in the mycobacteriology laboratory, a Ziehl- Neelsen staining is performed to confirm that it is an acidfast bacillus. In order to speciate, phenotypic characteristics such as colony morphology, growth rate, 
optimum growth temperature and photoreactivity are presumptively applied. More definitive test include biochemical tests and chromatographic techniques.

\section{BIOCHEMICAL TESTS}

These are batteries of tests that are based on the enzymatic and metabolic characteristics of the bacteria in terms of nutrient requirements and utilization as well as their metabolic products. For example MTB complex can be differentiated from the NTM by the following characteristics; buff colony on solid media, slow growth rate, Lack of pigment, reduction of nitrate, production of niacin and a positive catalase test which is inactivated at $68^{0} \mathrm{C}$.

\section{CHROMATOGRAPHY}

This technique is based on the difference in length of mycolic acid residue on the cell wall of different species. It is used for speciation following isolation by cultural techniques. This technique is used in reference laboratories for epidemiologic studies. Chromatographic techniques have changed as technology has evolved. Earlier methods such as column chromatography and thin layer chromatography have been replaced by gas chromatography and most recently by high pressure liquid chromatography

(HPLC).Chromatography is rapid, a total time of 2 hours is required. It is highly reproducible, and as little as a loopful of organism is required ${ }^{27}$. The draw back of this technique is the initial cost of equipment which is high.

The use of negative-ion mass spectrometry to detect tuberculostearic acid in clinical specimens is also gaining ground and hold promise for the rapid detection of mycobacteria ${ }^{28}$.

\section{GENOTYPING}

This is an essential tool in the laboratory for TB epidemiology. Genotyping relies on molecular techniques based on PCR. New PCRbased genotyping methods include Spacer Oligonucleotide Typing (Spoligotyping) and Mycobacterial Interspersed Repetitive Unit (MIRU) typing. Spoligotyping is based on differences between strains in the spacer sequences in the direct repeat locus of the $\mathrm{M}$. tuberculosis genome. MIRU is based on variable numbers of tandem repeat at 12 loci in the genome of $M$. tuberculosis ${ }^{29}$. These genotyping techniques can provide typing information from culture within a month from the time the specimen is collected from patient. Genotyping is useful in answering specific question related to suspected outbreak of TB in institutions such as hospitals schools and prisons. PCR based strain-typing requires only a small cell mass and yield numerical results that can be compared easily either within a laboratory or between laboratories to determine whether two strains are the same.

When used together for strain typing, spoligotyping and MIRU provide a powerful combination of tools that can discriminate strains which are not part of the chain of transmission ${ }^{21}$. Rarely, however, unrelated strain could have the same spoligotyping and MIRU number $^{21}$. This limitation has given rise to DNA fingerprinting

\section{DNA fingerprinting}

DNA fingerprinting based on restriction fragment length polymorphism (RFLP) is the gold standard for strain- typing in mycobacteriology. The insertion sequence IS6110 was specifically identified as the target of DNA probe to be used in fingerprint analysis ${ }^{30,31}$. It requires a week for the assay to be performed and results evaluated. The 
limitations of genotyping by RFLP is that a large cell mass is required and the results are band-pattern, thereby difficult to convert into digital format making comparison difficult. It is however available only in regional laboratories and not yet a routine technique.

\section{DRUG SUSCEPTIBILITY AND RESISTANCE TESTING}

The need for prompt and accurate antimycobacterial susceptibility testing for Mycobacteria tuberculosis has become a necessity, since the emergence of Multi Drug Resistant (MDR) strains. The different methods available include;

\section{PHENOTYPIC METHODS:}

These methods include proportional methods, the radiometric method, and modified phage amplification technique. In the proportional method, the mycobacteria isolate is incubated on two similar agar slope of Middlebrook $7 \mathrm{H} 10$. One of these contain a recommended concentration of antimycobacteria and the other free of the drug. This will require extra 2 weeks or more from the isolation of the organism. Interpretation is based on a standard proportion method which is the number of organisms resistant to each drug concentration as a percentage of the number of organism growing on the drug free media. Drug routinely tested are streptomycin, isoniazid, rifampicin and ethambutol. In the BACTEC Radiometric method, a standardized innoculum is inoculated into a drug free and a drug containing vial. The amounts and rates of $\mathrm{CO} 2$ produced in the absence and presence of the drug are compared to determine susceptibility or resistance. The BACTEC radiometric method would require extra 2 to 3 weeks from isolation. The BACTEK MGIT 960 and BACTEK 460 are currently the only methods currently cleared for use in the USA ${ }^{11}$. Susceptibility tests should be carried out in all initial isolates of $M$ tuberculosis and on isolates from patients who remain culture- positive after 3 months or demonstrate clinical evidence of therapeutic failure ${ }^{11}$.

In the modified phage amplification technique, the antimicrobial tested for is incorporated into the medium inoculated with the mycobacterium isolate, while the second medium is similarly inoculated without the drug. Growth comparison is made after incubation for 2 days.

\section{GENOTYPIC METHODS}

These entail molecular techniques aimed at detecting genes that code for resistance characteristics. Example includes; DNA probe and DNA sequencing of $M$. tuberculosis gene such as catalase (katG) or RNA polymerase (rpoB). Mutations in these genes have been associated with resistance to isoniazid and rifampicin respectively $^{32,33}$, the most common drugs used to treat TB. The use of Molecular beacons in a real- time PCR reaction, first described in 2004 , can differentiate between the presence of the wild- type sequence associated with drug susceptibility or a mutated sequence associated with drug resistance $^{34}$. Genotypic methods are not routinely used in the mycobacterium laboratory; they are essentially for research purposes

\section{THE FUTURE OF TB DIAGNOSTICS} The rapid technological evolution in the laboratory diagnosis of TB, especially in the application of molecular biology has diminished the time required for identification and susceptibility testing and may eliminate the need for lengthy culture and protracted biochemical methods. With continuous effort at increasing their reproducibility, ease of performance 
and cost containment, it is hoped that they will soon evolve from reference laboratories into clinical diagnostic laboratories. For now, there is need for integration of services provided by public and private laboratories with tuberculosis-control programmes to provide an effective system for diagnosis, treatment and control of $\mathrm{TB}^{35}$. In instances where some of the newer molecular methods are not available, or are not cost effective, as in developing countries, a system must be developed so that testing can be referred, in a timely manner to a reference laboratory. A critical component of such a system will be to assure that results are sent to the appropriate TB-control programmes, as well as to the health-care provider. Development and promotion of this integrated system is a challenge and responsibility of all mycobacteriology staff and public health experts.

\section{REFERENCES}

1. Hopewell PC. Impact of Human Immunodeficiency Virus Infection on the Clinical Features, Management and Control of Tuberculosis. Clin Infect Dis 1992; 15:540-547.

2. http://www.who.int/mediacentre/fact sheets/who104/en/ Assessed $2^{\text {nd }}$ June 2009.

3. Dolin PJ, Raviglione MC, Kochi A. Global Tuberculosis incidence and mortality during 1990-2000. Bull World Health Organ 1994. 72:213220.

4. Connie RM, Donald CL, George M, Textbook of Diagnostic Microbiology $3^{\text {rd }}$ Ed. Elsevier Publishing New Delhi India 2007: 683-715
5. Washinton W, Stephen A, William J et al. Koneman's Colour Atlas and Textbook of Diagnostic Microbiology; $6^{\text {th }}$ Ed. Lippincott Publishing. 2006; Chapter 19:10661117.

6. Krasnow I, Wayne L J, Comparison of Methods for Tuberculosis Laboratory. Appl Microbiol 1969; 28:915-917.

7. Kiehn TE, Edwards F, Brannon $P$, et al. Infection Caused by Mycobacterium avium complex in immunocompromised patients: Diagnosis by blood culture and feacal examination, antimicrobial susceptibility tests and morphological and seroagglutination characteristics. J Clin Microbiol 1985; 21:168-173.

8. Monica C,District Laboratory Practice in Tropical Countries,Part 1.Cambridge

Press, 1999: 39-40, 207-212.

9. Steingart $\mathrm{K}$, Henry $\mathrm{M}, \mathrm{Ng} \mathrm{V}$, et al. Fluorescence versus conventional sputum smear microscopy for tuberculosis: A systematic review. Lancet Infectious Disease 2006; 6(9):570-81.

10. Nolte FS, Metchock B. Mycobacterium. In: Murray PR, ed. Manual of Clinical Microbiology, $6^{\text {th }}$ Ed. Washington DC: ASM Press. 1995; Chapter 31.

11. Wood GL,Brown-Elliott BA, Desmond EP, et al. Susceptibility Testing of Mycobacteria,norcardiae, and other aerobic actinomycetes; Approved standard. NCCLS 2003; 23(18):168. 
12. Gill VJ, Park $\mathrm{CH}$, Stock F, et al. Use of Lysis-Centrifugation (Isolator) and radiometric (BACTEC) Blood Culture Systems for the Detection of Mycobacteria. J Clin Microbiol 1985; 22:543-546.

13. Macher AM, Kovacs JA, Gill V, et al. Bacteriaemia due to Mycobacterium intracellulare in acquired immunodeficiency syndrome. Ann Intern Med 1983; 99:782-785.

14. Chaturuedi N, Cockcroft A, Tuberculosis Screening Among Health Service Employees: Who Need X-ray? J. Soc. Occup Med 42:179Tuberculosis a Systematic Review. Lancet Infectious Disease 1992; 6(9): 570-81-82.

15. Menzies D, Pai M, Comstock G Meta analysis: New Tests for the Diagnosis of latent Tuberculosis Infection: Areas of Uncertainty and Recommendation for Research. Ann. Intern. Med. 2007; 146 (5):340-54.

16. Ferrara $G$ et al. Use in routine Clinical practice of two commercial blood test for diagnosis of infection with Mycobacterium tuberculosis: A prospective study. Lancet 2006; 367(9519):1328-1334.

17.Pai M, Riley LW, Colford JM Jr. Interferon-gamma assays in the immunodiagnosis of tuberculosis: a systemic review. Lancet Infect Dis.2004; 4:761-776.

18. Mon T, Sakatani M, Yamagishi F, etal. Specific detection of tuberculosis with an interferongamma using new antigen. Am J Respir Crit Care Med.2004; 170(1):59-64.
19. Kang YA, Lee HW, Yoon $\mathrm{H}$, et al. Discrepancy between the tuberculin skin test and the wholeblood interferon-gamma assay for the diagnosis of latent TB infection in an intermediate TB-burden country. JAMA 2005; 293:27562761.

20. Kang YA, Lee HW, Hwang SS, et al Usefulness of Whole Blood Interferon -gamma Assay and interferon -gamma EnzymeLinked immonospot Assay in the Diagnosis of Active Pulmonary Tuberculosis. Chest: 2007; 132 (3): 959-65.

21. Brown JW. TB. Keeping an ancient killer at bay; MLO 2004; 36(11):819.

22. Dinnes J, Deek J, Kurnst H, et al. A Systemic Review of Rapid Diagnostic Test for the Detection of Tuberculosis Infection, Health Technol Assess; 2007; 11(3):1314.

23. Crawford JT. New technologies in the diagnosis of tuberculosis. Semin Respir Infect.1994; 9:62-70.

24. Middleton AM,Chadwick MV, et al. Detection of Mycobacterium tuberculosis in mixed broth cultures using DNA Probe. Clin Microbiol Infec 1997; 3:668-671.

25. Reisner BS, Gatson AM, et al. Use of Gen Probe AccuProbes to identify Mycobacterium avium complex, Mycobacterium tuberculosis complex, Mycobacterium kansasii, and Mycobacterium gordonae directly from BACTEC TB broth culture. J Clin Microbiol 1994; 32:2995-2998. 
26. Tortoli E, Simonetti MT, et al. Evaluation of a commercial DNA probe assay for the identification of Mycobacterium kansasii. Eur J Clin Microbiol Infect Dis.1994; 13:264267.

27. Robert GD, Koneman EW, Kim YK. Mycobacterium. In: Balows A, ed. Manual of Clinical Microbiology $.5^{\text {th }}$ ed. Washinton DC; American Society for Microbiology.1991: Chapter 34.

28. Larsson AP, Odham G, Westerdahl $G$, Ollson B. Diagnosis of pulmonary tuberculosis by selected ion monitoring: improved analysis of tubreculosearate in sputum using negative-ion mass spectrometry. J Clin Microbiol 1987; 25:893-896.

29. Cave MD, Eisenach KD, McDermott PF et al. IS6110: Conservation of sequence in the Mycobacterium tuberculosis complex and its utilization in DNA fingerprinting. Mol Cell Probe 1991; 5:73-80.

30. Supply P. Mazars E, Lesjean S, et al. Variable human mini-satellite regions in $\mathrm{M}$. tuberculosis genome. Mol Microbiol 2000; 36:762-771.

31. Cave MD, Eisenach KD, Templeton G. Stability of DNA fingerprinting pattern produced with IS6110 in strains of Mycobacterium tuberculosis. J Clin Microbiol; 1994; 32:262-266.

32. Somoskovi A, Song $Q$ et al. Use of molecular methods to identify the Mycobacterium tuberculosis complex and other mycobacteria species and to detect rifampicin resistance in Mycobacterium tuberculosis complex isolates following growth detection with BACTEC MGIT 960 SYSTEM. J Clin Microbiol 2003; 41:2822-2826.

33. Viader-Salvado JM, Luna-Aguirre $\mathrm{CM}$ et al. Frequency of mutation on rpoB and codon 315 and 463 of katG in rifampicin-and isoniazidresistant Mycobacterium tuberculosis isolate from Northeast Mexico. Microb. Drug Resist.2003; 9:33-38.

34. Lin S-Y, Probert W, Desmond E. Rapid detection of isoniazid and rifampicin mutations in Mycobacterium tuberculosis complex from cultures or smearpositive sputa by use of molecular beacon. J Clin microbial. 2004; 42(9):4204-4208.

35. Masae K, Edward D. Tuberculosis and the expanding role of the laboratory. MLO 2005; 37(8):12-14. 